J. Phys. IV France 135 (2006) 141-142

(C) EDP Sciences, Les Ulis

DOI: $10.1051 / \mathrm{jp} 4: 2006135031$

\title{
Réalisation d'un laser à faible courant de seuil, avec des boites quantiques InAs/InP organisées et couplées latéralement
}

\author{
C. Cornet ${ }^{1}$, M. Hayne ${ }^{2}$, C. Levallois ${ }^{1}$, P. Caroff $^{1}$, E. Homeyer ${ }^{1}$, J. Even ${ }^{1}$, \\ C. Paranthoen ${ }^{1}$, H. Folliot ${ }^{1}$, C. Labbé ${ }^{1}$ et S. Loualiche ${ }^{1}$ \\ ${ }^{1}$ LENS-UMR FOTON 6082 au CNRS, INSA de Rennes, 20 avenue des Buttes de Coesmes, \\ 35043 Rennes Cedex, France \\ ${ }^{2}$ Laboratorium voor Vaste-Stoffysica en Magnetisme, Katholieke Universiteit Leuven, \\ Celestijnenlan 200D, 3001 Leuven, Belgium \\ e-mail : charles.cornet@ens.insa-rennes.fr
}

\begin{abstract}
Résumé. Nous présentons ici la réalisation d'un laser à faible courant de seuil avec des boites quantiques (QDs) organisées et couplées InAs/InP sur subsstrat (311)B pour une émission à $1.55 \mu \mathrm{m}$. En effet, pour des hautes densités de QDs, une organisation périodique apparaît dans le plan. Cette organisation renforce le couplage latéral inter-boites. Des expériences de magnéto-photoluminescence permettent de mettre en évidence ces effets de couplage. Ce couplage améliore l'injection des porteurs. Une émission laser avec des faibles courants de seuil est obtenue avec de telles boites.
\end{abstract}

\section{INTRODUCTION}

Depuis quelques années, les structures de basse dimensionnalité suscitent un intérêt grandissant, du fait de leurs prometteuses propriétés optoélectroniques [1]. Les boîtes quantiques (QDs), objets possédant un confinement quantique dans les trois directions de l'espace laissent d'ores et déjà espérer une amélioration des performances des composants lasers [1]. L'objet de ce travail concerne l'étude des propriétés optiques des QDs InAs/InP sur substrat (311)B, émettant à $1.55 \mu \mathrm{m}$, longueur d'onde des télécommunications optiques, pour l'amélioration des performances des lasers. Nous montrons ici que l'utilisation de hautes densités de boites engendre un couplage entre boites. Ce couplage est mis en évidence par des expériences de magnéto-photoluminescence. Il facilite l'injection des porteurs dans les boites. Une émission laser avec un faible courant de seuil est obtenue dans ce système.

\section{BOITES QUANTIQUES ORGANISÉES}

De récentes études ont montré qu'il était possible d'atteindre des hautes densités de QDs (jusqu'à $1,6.10^{11} \mathrm{~cm}^{-2}$ ) dans le système InAs/InP. Au delà d'une certaine densité de boites, une organisation périodique apparaît suivant des directions cristallines particulières (Fig. 1 (a)). Des calculs préliminaires ont déjà montré l'influence du couplage sur les propriétés électroniques de nos boites [2]. Pour de telles densités, et en présence d'un ordre apparent, un couplage latéral électronique devait apparaître, engendrant des effets de séparation et de décalage des niveaux d'énergie de nos QDs [3].

\section{BOITES QUANTIQUES COUPLÉS ET EMISSION LASER}

Afin de mettre en évidence cet effet de couplage latéral, des expériences de magnéto-photoluminescence [4] ont été réalisées sur deux échantillons avec des densités de boites différentes. Alors que les images AFM montrent que pour de fortes densités de boites, les rayons des boites deviennent plus petits, les mesures en champ magnétique montrent que le rayon de l'exciton dans ces boites devient plus grand 

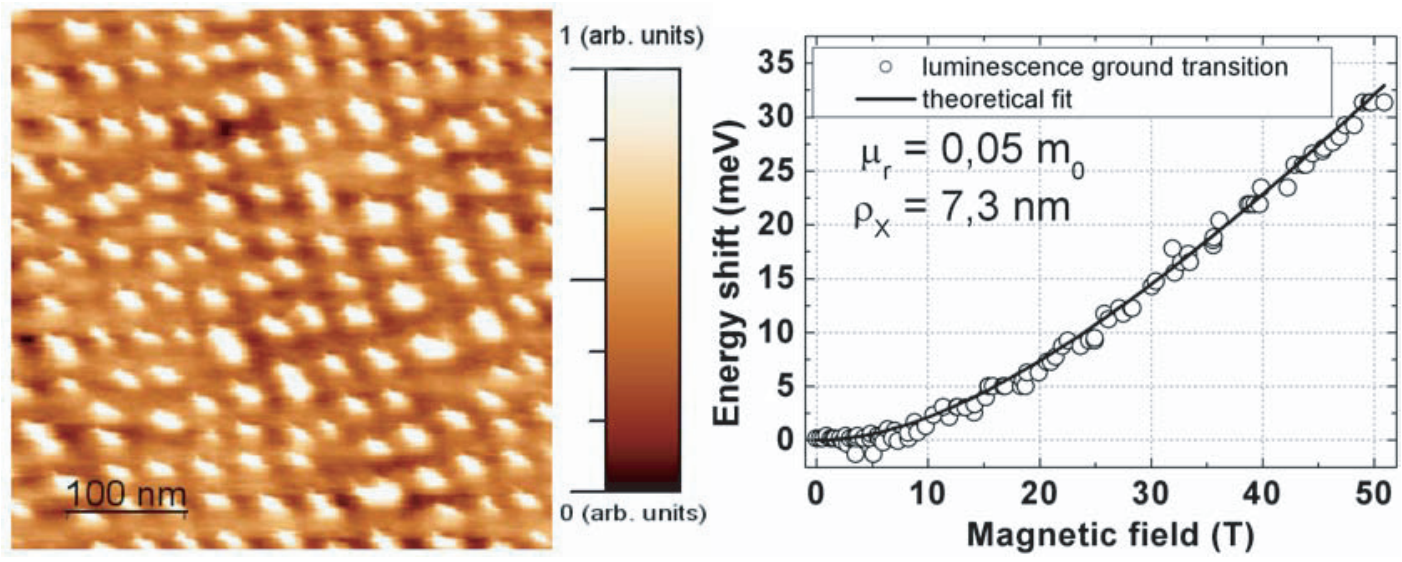

Figure 1. Image AFM des boites quantiques couplées (a). La magnéto-photoluminescence est utilisée pour démontrer l'existence du couplage latéral, permettant de réduire ainsi les courants de seuil de nos lasers (b).

(Fig. 1 (b)). La fonction d'onde est donc délocalisée pour les hautes densités de boites à cause du couplage latéral. Cet effet de couplage doit ainsi permettre une meilleur redistribution des porteurs dans les boites. Il doit ainsi pouvoir abaisser sensiblement les courants de seuils de nos lasers. Deux échantillons lasers ont été conçus avec des hautes et basses densités [5]. Les courants de seuil mesurés sont respectivement $190 \mathrm{~A} . \mathrm{cm}^{-2}$ et $420 \mathrm{~A} . \mathrm{cm}^{-2}$. Nous interprétons cette différence comme étant due au couplage entre boites.

\section{Remerciements}

Les auteurs tiennent à remercier le programme EUROMAGNET ainsi que le réseau d'excellence européen SANDIE pour leur soutien financier à ces travaux de recherche.

\section{Références}

[1] D. Bimberg, M. Grundmann and N. N. Ledentsov, Quantum Dot Heterostructure (John Whiley \& Sons Ltd, Chichester, England, 1999).

[2] P. Miska, J. Even, C. Paranthoen, O. Dehaese, A. Jbeli, M. Senès and X. Marie, Appl. Phys. Lett. 86, 111905 (2005).

[3] C. Cornet, C. Platz, P. Caroff, J. Even, C. Labbé, H. Folliot, A. Le Corre and S. Loualiche, Phys. Rev. B, vol. 72, 035342 (2005).

[4] M. Hayne, J. Maes, S. Bersier, M. Henini, L. Müller-Kirsch, R. Heitz, D. Bimberg and V. V. Moshchalkov, Physica B 346, 421 (2004).

[5] C. Platz, C. Paranthoen, P. Caroff, N. Bertru, C. Labbé, J. Even, H. Folliot, A. Le Corre, S. Loualiche, G. Moreau, J. C. Simon and A. Ramdane, Semicond. Sci. Technol. 20, 459 (2005). 